\title{
KNOTS WITH HEEGAARD GENUS 2 COMPLEMENTS ARE INVERTIBLE
}

\author{
RICHARD P. OSBORNE
}

\begin{abstract}
Let $K$ be a polyhedral oriented knot in $S^{3}$ and $N(K)$ be a regular neighborhood of $K$. If $S^{3} \sim \dot{N}(K)$ can be constructed by attaching a single 2-handle to a genus two handlebody, then there is a homeomorphism of $S^{3}$ onto itself mapping $K$ onto itself and reversing the orientation of $K$.
\end{abstract}

We prove the title. A somewhat more careful statement is the following.

ThEOREM. Let $K \subset S^{3}$ be a polyhedral knot and let $N(K)$ be a regular neighborhood of $K$. If $S^{3} \sim \stackrel{\circ}{N}(K)$ can be constructed by attaching a single 2-handle to a genus two handlebody, then $K$ is invertible.

Proof. By a meridian of the knot $K$ we mean a polyhedral disk $D$ in $N(K)$ with $\partial D \subset \partial N(K)$ and $N(K) \sim N(D)$ is homeomorphic with a ball. $(N(D)$ is a regular neighborhood of $D$ in $N(K)$.) We show how to construct a self-homeomorphism of $S^{3} \sim \stackrel{N}{N}(K)$ which maps the boundary of a meridian of the knot onto its inverse. It is easy to see that such a homeomorphism can be extended to an involution of $S^{3}$ taking the (oriented) knot to its inverse. Let $\mathrm{H}_{2}$ be a genus two solid handlebody, let $D_{1}$ and $D_{2}$ be meridian disks for $H_{2}$ and let $\gamma$ be the simple closed curve on $H_{2}$ to which a 2-handle $B$ is attached to get $S^{3} \sim N(K)$. Let $m$ be the boundary of a meridian of $K$. Without loss of generality we may assume that $m \subset \partial H_{2} \sim \gamma$. Let $h: H_{2} \rightarrow H_{2}$ be a rotation of $H_{2}$ through $180^{\circ}$ about its axis. (Think of the standard picture of $\mathrm{H}_{2}$. The axis passes through both holes of the handlebody.) Now $h$ induces the symmetry $\eta$ as defined in [O \& S, p. 248]. Thus $h$ can be assumed to map $\gamma$ and $m$ onto themselves while reversing their orientations (see also [B \& H, \&5]). The underlying reason for this is that the rotation inverts the Lickorish twists that generate the homeotopy group of the surface. Clearly then $h$ may be extended to a homeomorphism $\tilde{h}$ which maps $B$ onto itself while reversing orientation. This completes our proof.

Note. This result shows that knots such as $8_{20}$ and $10_{132}$ from the table of knots in [Rolf] are invertible. This does not appear evident from the presentations given. These knots are not torus knots or 2-bridge knots. The knot $8_{10}$ does not have a complement with Heegaard genus 2 but it is invertible. That it does not have such a complement follows from the fact that its second elementary ideal is proper [Fox]. Of course, the result above means that the noninvertible pretzel knots of Trotter

Received by the editors February 18, 1980 and, in revised form, April 21,1980.

1980 Mathematics Subject Classification. Primary 57M25. 
[Trot] all have complements of Heegaard genus 3 . It is certainly not easy to decide whether a given knot has a complement of Heegaard genus 2 .

\section{REFERENCES}

[O \& S] R. P. Osborne and R. S. Stevens, Group presentations corresponding to spines of 3-manifolds. III, Trans. Amer. Math. Soc. 234 (1977), 245-251.

[B \& H] J. S. Birman and H. M. Hilden, Heegaard splittings of branched coverings of $S^{3}$, Trans. Amer. Math. Soc. 213 (1974), 315-352.

[Rolf] D. Rolfsen, Knots and links, Publish or Perish, Cambridge, Mass., 1976.

[Fox] R. H. Fox, A quick trip through knot theory, Topology of 3-Manifolds, M. K. Fort, Jr., ed., Prentice-Hall, Englewood Cliffs, N. J., 1962.

[Trot] H. F. Trotter. Non-invertible knots exist, Topology 2 (1964), 275-280.

Department of Mathematics, Colorado State University, Fort Collins, Colorado 80523 